Electronic version of an article published as [Polymer, 2008, vol.49, No.10, p. 24402443] [DOI:10.1016/j.polymer.2008.03.037]

(C) [copyright Elsevier] 


\title{
Study of dispersive mobility in polyimide by surface voltage decay measurements
}

\author{
A. Aragoneses * M. Mudarra J. Belana J. A. Diego \\ Dept. Física i Enginyeria Nuclear. ETSEIAT \\ C. Colon, 11 Terrassa 08222, Barcelona, Spain
}

\begin{abstract}
In order to study charge trapping and transport in polyimide, we have submitted samples of Kapton HN to corona charging and measured its surface potential decay with an electrostatic voltmeter. We propose a two terms mobility to explain the experimental data: a non dispersive contribution based on Toomer and Lewis model added to a dispersive process, which is associated with the disordered structure of the material. The non-dispersive model alone did not fit well to the data for short times, but our assumption makes the theoretical expresion fit succesfully to the experimental data. Some important parameters related to the charge transport properties of the material are determined and discussed.
\end{abstract}

Key words:

conductivity, polyimide, dispersive conductivity, space charge, charge decay

PACS: 72.10.Bg, 72.20.jv, 72.80.Ng, 73.50.-h

* All correspondence should be addressed to A. Aragoneses
Email address: andres.aragoneses@upc.edu (A. Aragoneses).

Preprint submitted to Polymer

5 November 2007 


\section{INTRODUCTION.}

Polyimide (PI) has become widespread in aerospace industry because of its thermal and electrical insulating properties. Due to its properties, this material is used as a thermal blanket to prevent artificial satellites systems from wide temperature oscillations. Satellites in space environment are exposed to intense radiations (energetic electrons, ions, X-rays, ...) that charge electrically its surface. Polyimide, as it happens in other insulating materials, gets electrically charged when it is subjected to radiation, so that spontaneous discharges may arise that can lead to malfunctions of the sensitive systems on board[1-4]. Some studies on PI can be found in the literature that deal with new synthesis processes [5] and fabrication of composites with carbon nanotubes [6] and with silica [7] in order to improve its mechanical and electrical properties.

There have been registered a few cases where normal operations of spacecraft electronics have been interrupted related to this spacecraft charging[8]. Understanding and controlling the processes that lead to space charge formation and relaxation in this material are, thus, relevant in order to improve the performance of such blankets. The aim of this paper is to study the space charge relaxation process in polyimide, in order to characterize the microscopic processes that contribute to the mobility of the carriers.

Surface potential decay experiments are widely used as it is an efficient method in the study of charge transport in polymers [9-16]. In these experiments a film of polymer is submitted to a discharge from a needle and the ions produced are placed on the surface of the film. Because of their own electric field, it takes place electron or hole transport towards the rear electrode in order to 
reach electrical equilibrium. Several authors have tried to describe the way the samples discharge [17-21]. Carriers in this situation do not move with the proper mobility of the band, but it is modulated because of the traps and because of the presence of other carriers. That is why an effective mobility $\mu_{e f f}$ has to be considered

$$
\mu_{e f f}(t)=\mu_{0} \eta(t)
$$

Here $\mu_{0}$ is the proper mobility in the band and $\eta(t)$ is the ratio of carriers contributing to the process at a time $t$.

\section{THEORY.}

In order to explain the surface potential decay observed in PI we shall consider that charge transport through the sample is due to two contributions. Once the sample has been charged, a fraction of the carriers follows a non-dispersive transport while the other fraction follows a dispersive transport. That is why we shall consider a non-dispersive term together with a dispersive one. As charges travel through the sample they are being trapped and only a small portion of them is contributing to mobility. This lowers the density of charges in further positions so there are less charges competing for the traps and the effective correlation diminishes, that is why we can expect the dispersive term to be more important for short times. Taking this into account we write the effective mobility as:

$$
\mu_{e f f}(t)=\mu_{N}(t)+\mu_{D}(t)=\mu_{0}\left(\eta_{N}(t)+\eta_{D}(t)\right)
$$


where subindex $N$ refers to the non-dispersive process and $D$ to the dispersive one respectively.

\subsection{Non-dispersive mobility.}

In order to deduce the expression of the modulated mobility for the nondispersive contribution, we consider that charges, after the corona injection, are injected into the bulk instantaneously. The band structure can provide several kinds of traps for the charge carriers, with different energetic depths. The initial charge spreads into the different traps, being $a_{i}$ the portion of the initial charge trapped in the state $i=1 \ldots n$, with $\sum_{i=1}^{n} a_{i}=1$. Charges drift through the sample hopping from one localized state to another, being $r_{r i}$ the probability per unit time of a charge to release from a trapping level $i$ and $r_{t i}$ the probability per unit time to be retrapped at trapping level $i$. In this case the function $\eta(t)$ has the form [22]

$$
\eta_{N}(t)=\mathrm{LT}^{-1}\left[\frac{1}{s} \frac{1}{1+\sum_{i=1}^{n} \frac{r_{t i}}{s+r_{r i}} a_{i}}\right]
$$

where LT refers to the Laplace transform of the function and $s$ is the reciprocal time.

In order to ensure our assumption that all charges are injected instantaneously, we will apply a high enough electric field to the needle. In this case charges do not fall into surface traps from where they jump to the bulk, but are directly injected into the bulk, where the dynamics of charge transport occurs by hopping between localized trapping centers. In our study we consider the simplest case: we assume the existence of one trapping level thermally connected and that thermally disconnected levels are filled. Under these considerations, (3) 
can be written

$$
\eta_{N}(t)=\mathrm{LT}^{-1}\left[\frac{1}{s} \frac{1}{1+\frac{r_{t}}{s+r_{r}}}\right]
$$

Transformation of (4) to time domain leads to

$$
\eta_{N}(t)=\frac{r_{r}+r_{t} \mathrm{e}^{-R t}}{R} \quad\left(R=r_{r}+r_{t}\right)
$$

This expression was previously found by Toomer and Lewis[19] considering the dinamics of the hopping transport, defining

$$
\mu_{e f f}\left(n_{f}+n_{t}\right)=\mu_{0} n_{f}
$$

where $n_{t}$ refers to the trapped charge and $n_{f}$ refers to the free charge, the one that contributes to mobility.

When charges get the rear, grounded electrode they do not contribute any more to the surface potential that we measure, so the decay behaves different during the travel of the leading charges and after they have reached the grounded electrode. The time this front of charges takes to get through the sample is the transit time, $t_{\tau}$, We shall limit our study to $t<t_{\tau}$. Up to the transit time the derivative of the surface potential can be written [19]:

$$
\frac{\mathrm{d} V}{\mathrm{~d} t}=-\frac{1}{2} \mu_{e f f}(t) \frac{V_{0}^{2}}{d^{2}}
$$

where $d$ is the sample thickness. 


\subsection{Dispersive mobility.}

Several authors[22-27] have treated dispersive mobility theoretically but, as far as we know, low attention has been paid from the experimental point of view. This behaviour has been taken on account by several authors in order to describe the anomalous transport observed in some photosensitive materials, and has been extended succesfully to different areas such as fluid dymanics, geology, brownian movement, as well as to electric transport in insulating materials[27-30].

In order to explain charge transport in disordered materials, Scher and Montroll [24] developed a stochastic transport model. This model is based on the dispersion that appears in these materials in the distances between localized sites available for carriers to hop and on the dispersion of the potential barriers between these sites. This affects the hopping time of charges, the arriving time from site to site. These charges are supposed to follow a time dependent random walk governed by a waiting-time distribution function $\psi(t)$. The shape of this distribution function will be related to the lack of order of the material. In the reciprocal time domain this function is related to $\eta(t)$ by $[24,25]$

$$
\operatorname{LT}[\eta(s)]=\frac{s \operatorname{LT}[\psi(s)]}{1-\operatorname{LT}[\psi(s)]}
$$

Charge transport in a free of traps ordered material is described by a Gaussian distribution function $\psi(t) \propto e^{-\beta t}$, where the packet of charges does not disperse and the shape of the distribution mantains. This corresponds to a constant mobility, $\mu \propto \mu_{0} \delta(t)$. But a dispersive transport regime in a potential decay process is characterized[24] by $\psi(t) \propto t^{-1-\alpha}$, which gives a time 
dependence of mobility as

$$
\eta(t) \approx \mathrm{LT}^{-1}\left[k s^{1-\alpha}\right]
$$

where $k$ is a constant and $0 \leq \alpha \leq 1$. For the initial stage of the discharge, the time derivative of the surface potential is approximately [25]:

$$
\frac{\mathrm{d} V}{\mathrm{~d} t} \approx-\frac{k}{2 \Gamma(\alpha)} \frac{V_{0}^{2}}{d^{2}} t^{\alpha-1}
$$

From this equation $\eta_{D}(t)=\gamma t^{\alpha-1}$, where $\gamma$ is a parameter that depends on $\alpha$, $k$ and the proper mobility $\mu_{0}$.

This new term, together with the non-dispersive one, results in an effective mobility as

$$
\mu_{e f f}=\mu_{0}\left(\frac{r_{r}+r_{t} \mathrm{e}^{-R t}}{R}+\gamma t^{\alpha-1}\right)
$$

After integration of equation(7) with the effective mobility given by (11) the surface potential of the sample can then be written

$$
V(t)=V_{0}\left(1-\frac{\mu_{0}}{2 R} \frac{V_{0}}{d^{2}}\left(r_{r} t+\frac{r_{t}}{R}\left(1-\mathrm{e}^{-R t}\right)\right)-\lambda t^{\alpha}\right)
$$

where

$$
\lambda=\frac{\mu_{0} V_{0} \gamma}{2 d^{2} \alpha}
$$

\section{EXPERIMENTAL.}

To check the validity of our assumption we have charged several samples of Kapton HN of $50.8 \mu \mathrm{m}$ thick via the corona triode [?]. The samples were 
one side metalized. They were charged at different initial potentials at the non-metalized side. In less than one second after being charged, the surface potential was being measured with a Trek model 347 electrostatic voltmeter. Kapton samples were provided by Dupont and were kept at a temperature between $22^{\circ} \mathrm{C}$ and $25^{\circ} \mathrm{C}$ and in an ambiance of relative humidity between $40 \%$ and $50 \%$ from when received and during the experiments. The influence of elevated humidity on the electrical conductivity of polyimide can be found in the literature[32,33]. The experiments were carried out in a dark environment. The needle of the corona triode was at $15 \mathrm{kV}$ during the charging process in order to ensure injection. In Figures 2 and 1 we show in a lin-log scale the normalized potential versus time for the different samples, positive and negative and the corresponding experimental points.

\section{RESULTS AND DISCUSSION.}

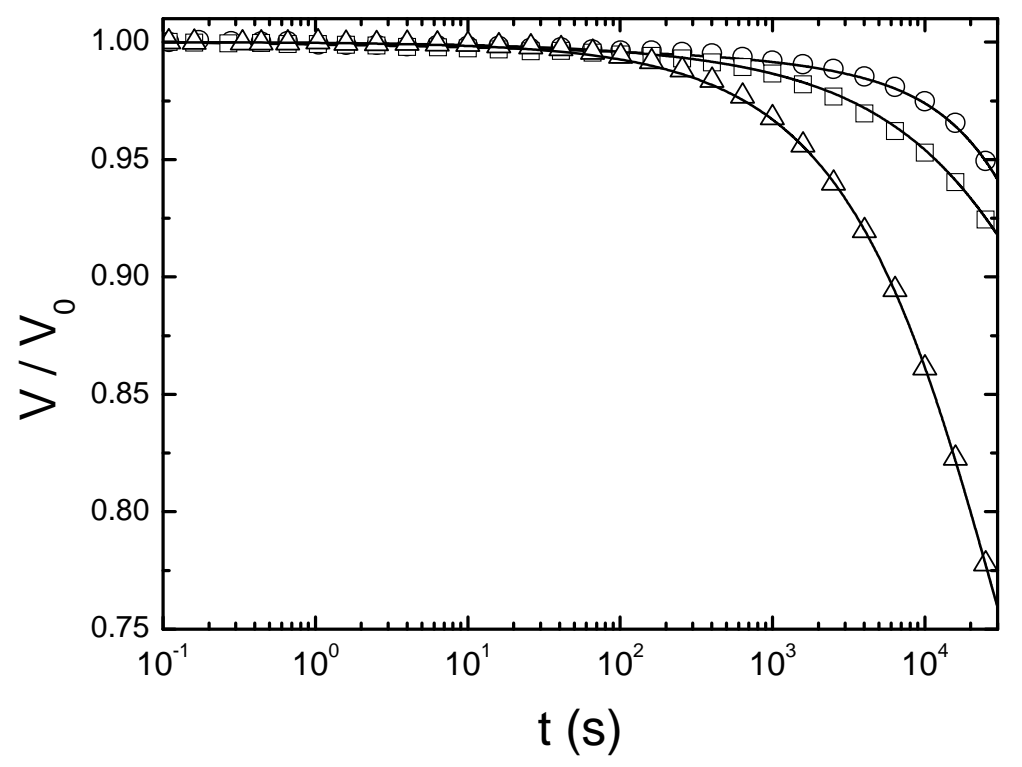

Fig. 1. Normalized surface potential versus time for negative initial potentials. $\bigcirc-1000 \mathrm{~V}, \square-2000 \mathrm{~V}, \Delta-3000 \mathrm{~V}$. Lines are the theoretical fit after equation (12). 
We can appreciate the accuracy of the fit in Figures 1 and 2, achieved taking on account both terms. Were the dispersive term not considered, the slope predicted by the model at the initial step of the charge decay would be lower than experimentally observed (not shown). So that both mechanisms, dispersive and non dispersive, are necessary to describe the potential decay from the initial stage up to the transit time accurately.

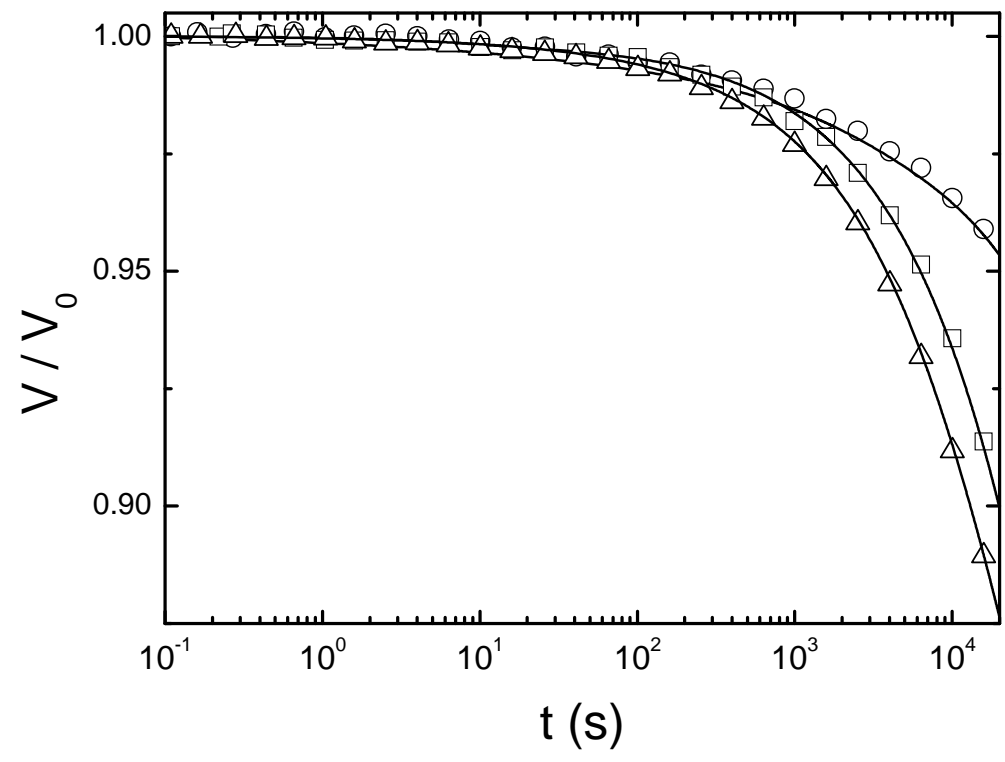

Fig. 2. Normalized surface potential versus time for positive initial potentials. $\bigcirc 1000 \mathrm{~V}, \square 2000 \mathrm{~V}, \triangle 3000 \mathrm{~V}$. Lines are the theoretical fit after equation (12).

In Table 1 the different free parameters resulting from fitting (12) to experimental data are showed. After the values for the intrinsic band mobility we see they do not depend on the initial potential significantly, for the studied values, and they do not depend on the sign of the charge carriers. The small differences do not seem significant, although a mean value of the mobility for negative carriers appears slightly smaller $\left(2.06 \times 10^{-17} \mathrm{~m}^{2} \mathrm{~V}^{-1} \mathrm{~s}^{-1}\right)$ than for positive carriers $\left(2.37 \times 10^{-17} \mathrm{~m}^{2} \mathrm{~V}^{-1} \mathrm{~s}^{-1}\right)$.

After a look at the normalized plots we observe that the potential decay is faster with the higher initial potential. This seems reasonable as a non 
Table 1

Parameters of the model calculated fitting the experimental data to equation (12).

\begin{tabular}{rrrr|rr}
$V_{0}(\mathrm{~V})$ & $\mu_{0}\left(\times 10^{-17} \mathrm{~m}^{2} \mathrm{~V}^{-1} \mathrm{~s}^{-1}\right)$ & $r_{r}\left(\mathrm{~s}^{-1}\right)$ & $r_{t}\left(\mathrm{~s}^{-1}\right)$ & $\lambda\left(\mathrm{s}^{-\alpha}\right)$ & $\alpha$ \\
\hline 976 & 2.208 & $1.61 \times 10^{-4}$ & $8.79 \times 10^{-3}$ & $1.15 \times 10^{-3}$ & 0.367 \\
1814 & 2.779 & $1.83 \times 10^{-4}$ & $3.43 \times 10^{-4}$ & $1.21 \times 10^{-3}$ & 0.295 \\
2797 & 2.126 & $9.48 \times 10^{-5}$ & $2.10 \times 10^{-4}$ & $1.54 \times 10^{-3}$ & 0.313 \\
\hline-983 & 2.025 & $4.63 \times 10^{-4}$ & $6.36 \times 10^{-4}$ & $4.83 \times 10^{-3}$ & 0.094 \\
-2018 & 2.020 & & & & \\
-3011 & 2.133 & $4.01 \times 10^{-5}$ & $2.34 \times 10^{-4}$ & $3.93 \times 10^{-3}$ & 0.129 \\
& & & & &
\end{tabular}

field dependent mobility has been assumed and a higher field results in a higher carrier drift velocity. The parameter $r_{r}$ decreases when the initial field is increased, so it seems more difficult for carriers to scape from traps when a higher initial potential is applied. As we are working for relatively short times (a time scale smaller than the average time a carrier remains trapped, $r_{r}^{-1}$ ) this lowering indicates that charges get trapped deeper for higher fields. On the other hand $r_{t}$ seems to lower with the rising initial surface potential, although for $-3000 \mathrm{~V}$ it reverses this trend. We related this behavior to a lowering of the potential barriers of the traps associated with the field, that results in a decrease of the effective trapping.

The parameter $\alpha$ can be associated with the sublinear power-law observed in conductivity in the frequency domain [34-37]. This power-law conduction regime is associated with the slowing down of the relaxation process in the frequency domain as a result of the cooperative effects, in the same way as the 
KWW function does in the time domain. An important connection between these two approaches stems from the coupling model of Ngai and Kannert[38]. This model predicts a power-law conductivity associated with the KWW relaxation function due to the correlation among charge carriers.

The values of $\alpha$ obtained depend on the sign of the carrier (Table 1). For negative carriers this contribution grows with the initial surface potential, showing that the more carriers in the sample the higher correlation among them. But for positive carriers we do not appreciate a significative dependence with the applied field. This may be related to differences in transport properties between electrons and holes.

\section{CONCLUSIONS.}

We have seen that, in order to explain charge decay in Kapton HN polyimide, previous models do not fit well for the first seconds of the decay. They behave quite properly for longer times, but for short times their decay is almost horizontal on lin-log scales, when the experimental plot happens to have a higher slope. It is only taking into account an additional, dispersive behavior, that the theoretical expression proposed for the potential decay improves the accuracy of the fit. Results also show different behaviour for positive and negative charge carriers; while dispersive parameters do not manifest strong changes for positive charges (holes in the conduction band), they do change significantly for negative charges (electrons in the valence band). 


\section{ACKNOWLEDGMENTS}

This research work has been partially supported by Spanish government project PTR95.0742.OP.

\section{References}

[1] S. T. Lai, Spacecraft charging thershold in single and double maxwellian space environmnets., IEEE Trans. on Nucl. Sci. 6 (1991) 1629-1634.

[2] A. R. Frederickson, Upsets related to spacecraft charging, IEEE Trans. on Nucl. Sci. 2 (1996) 426-441.

[3] H. B. Garret, A. C. Whittlesey, Spacecraft charging, an update, IEEE Trans. on Pasma Phys. 6 (2000) 2017-2028.

[4] A. R. Frederickson, C. E. Benson, J. F. Bockman, Measurement of charge storage and leakage in polyimides, Nucl. Instr. and Meth. in Phys. Res. B 208 (2003) 454-460.

[5] Y. Yin, J. Fang, Y. Cui, H. K. K. Tanaka, K. Okamoto, Synthesis, proton conductivity and methanol permeability of a novel sulfonated polyimide from 3-(2',4'-diaminophenoxy)propane sulfonic acid, Polymer 44 (2003) 4509-4518.

[6] X. Jiang, Y. Bin, M. Matsuo, Electrical and mechanical properties of polyimidecarbon nanotubes composites fabricated by in situ polymerization, Polymer 46 (2005) 7418-7424.

[7] P. Musto, G. Ragosta, G. Scarinzi, L. Mascia, Polyimide-silica nanocomposites: spectroscopic, morphological and mechanical investigations, Polymer 45 (2004) $1697-1706$. 
[8] D. N. Baker, The occurence of operational anomalies in spacecraft and their relationship to space weather, IEEE Trans. Plas. Sci. 28 (2000) 2007-2016.

[9] J. K. Quamara, P. K. C. Pillai, B. L. Sharma, Surface potential decay characteristics and tsdc studies in corona charged kapton polyimide film., Acta Polimerica 34 (1983) 265-267.

[10] R. Coelho, L. Levy, D. Sarrail, On the natural decay of corona charged insulating sheets., Phys. stat. sol. (a) 94 (1986) 289-298.

[11] L. Centurioni, G. Coletti, F. Guastavino, An experimental study to investigate the effects of partial discharges (pd) of electric field and of relative humidity during surface pd tests on thin polymer films., Conference on Electrical Insulation and Dielectric Phenomena. 0 (1999) 239-242.

[12] L. Centurioni, F. Guastavino, P. Tiemblo, G. M. Yang, G. M. Sessler, Charge decay on polymers subjected to ageing by partial discharges., Polymer Int. 46 (1998) 47-53.

[13] L. Centurioni, F. Guastavino, P. Tiemblo, G. M. Yang, G. M. Sessler, Charge decay on polymers subjected to ageing by partial discharges., Polymer Int. 46 (1998) 47-53.

[14] G. Chen, H. Xiao, C. Zhu, Charge dynamic characteristics in corona-charged polytetrafluoroethylene film electrets, Journal of Zhejiang University Science 8 (2004) 923-927.

[15] P. Llovera, P. Molinié, New methodology for surface potential decay measurements: application to study charge injection dynamics on polypropylene films., IEEE Trans. Diel. Electr. Insul. 11 (2004) 1049-1056.

[16] G. Chen, Z. Xu, L. W. Zhang, Measurement of the surface potential decay of corona-charged polymer films using the pulsed electroacoustic method, Meas. Sci. Technol. 18 (2007) 1453-1358. 
[17] H. J. Wintle, Surface-charge decay in insulators with nonconstant mobility and deep trapping, J. Appl. Phys. 43 (1972) 2927-2930.

[18] T. J. Sonnonstine, M. M. Perlman, Surface-potential decay in insulators with field-dependent mobility and injection efficiency, J. Appl. Phys. 46 (1975) 39753981.

[19] R. Toomer, T. J. Lewis, Charge trapping in corona-charged polyethylene films, J. Phys. D: Appl. Phys. 13 (1980) 1343-1356.

[20] M. Campos, J. A. Giacometti, Surface-potential decay in insulators with deep traps, J. Appl. Phys. 52 (1981) 4546-4552.

[21] H. von Berlepsh, Interpretations of surface potential kinetics in hdpe by a trapping model, J. Phys. D: Appl. Phys. 18 (1985) 1155-1170.

[22] F. Mady, R. Renoud, J.-P. Ganachaud, J. Bigarré, Potential decay experiments for the characterization of electron transport. modelling and discussion, Phys. Stat. Sol. (b) 242 (2005) 2089-2106.

[23] M. F. Shlesinger, Asymptotic solutions of continuous-time random walks, Journal Stat. Phys. 10 (1974) 421-434.

[24] H. Scher, E. W. Montroll, Anomalous transit-time dispersion in amorphous solids, Phys. Rev. B 12 (1975) 2455-2477.

[25] G. F. L. Ferreira, L. E. Carrano, Space-charge transport in disordered media, Phys. Rev. B 56 (1997) 11579-11583.

[26] K. W. Kehr, K. P. N. Murthy, H. Ambaye, Connection between dispersive transport and statistics of extreme events, Physics A 253 (1998) 9-22.

[27] A. Picos-Vega, R. Ramírez-Bon, Transient transport in disordered multilayers., Phys. Rev. B 64 (2001) 142011-142015. 
[28] B. Berkowitz, H. Scher, Anomalous transport in random fracture networks, Phys. Rev. Lett. 79 (1997) 4038-4041.

[29] M. F. Shlesinger, J. Klafter, G. Zumofen, Above, below and beyond brownian motion., Am. J. Phys. 67 (1999) 1253-1259.

[30] B.Berkowitz, H. Scher, S. E. Silliman, Anomalous transport in laboratosy-scale, heterogeneous porous media., Water Resources Research 36 (2000) 149-158.

[31] C. J. Dias, J. N. Marat-Mendes, J. A. Giacometti, Effects of a corona discharge on the charge stability of teflon fep negative electrets, J. Phys. D: Appl. Phys. 22 (1989) 663-669.

[32] F. Belluci, I. Khamis, S. D. Senturia, R. M. Latanision, Moisture effects on the electrical conductivity of kapton polyimide, J. Electrochem. Soc. 137 (1990) $1778-1784$.

[33] N. A. Galichin, M. E. Borisova, The influence of elevated humidity on the stability of the electret state in polyimide films, Russian Electrical Engineering 78 (2007) 129-132.

[34] M. F. Shlesinger, E. W. Montroll, On the williams-watts function of dielectric relaxation., Proc. Natl. Acad. Sci. USA 81 (1984) 1280-1283.

[35] A. V. Milovanov, K. Rypdal, Streched exponential relaxation and ac universality in disordered dielectrics., Phys. Rev. B 76 (2007) 1042011-1042018.

[36] M. Mudarra, J. Belana, J. C. Cañadas, J. A. Diego, J. Sellarès, Space charge relaxation in polyetherimides by electric modulus formalism., J. Apl. Phys. 88 (2000) 4807-4812.

[37] M. Mudarra, R. Díaz-Calleja, J. Belana, J. C. Cañadas, J. A. Diego, J. Sellarès, M. J. Sanchis, Sublinear dispersive conductrivity in poly(methyl methacrylate at temperatures above the glass transition, Polymer 45 (2004) 2737-2742. 
[38] D. L. Sidebottom, P. F. Green, R. K. Brow, Two contributions to the ac conductivity of alkali oxide glasses, Phys. Rev. Lett. 74 (1995) 5068-5071. 